\title{
Overall Distribution Pattern of the Intramuscular Nerves of the Extraocular Muscles and its Clinical Implications
}

\author{
Patrón de Distribución General de los Nervios Intramusculares \\ de los Músculos Extraoculares y sus Implicaciones Clínicas
}

Guoyan Zhou; Jiayu Zhou; Danli Wang \& Shengbo Yang

ZHOU, G.; ZHOU, J.; WANG, D. \& YANG, S. Overall distribution pattern of the intramuscular nerves of the extraocular muscles and its clinical implications. Int. J. Morphol., 39(5):1412-1419, 2021.

SUMMARY: The purpose of this study was to reveal the overall distribution pattern of the intramuscular nerves of each extraocular muscle and provide morphological guidance for the selection of the neuromuscular compartment during extraocular muscle transplantation and target localization of the botulinum toxin A injection to correct strabismus. We studied 12 Chinese head specimens that were fixed with formalin. The extraocular muscles from both sides of each head were removed, and a modified Sihler's staining technique was used to reveal the overall distribution pattern of the intramuscular nerves. We observed an intramuscular nerve-dense region formed by the intramuscular arborized branches in the semitransparent superior rectus, inferior rectus, medial rectus, lateral rectus, superior oblique, inferior oblique, and levator palpebrae superioris muscles with Sihler's staining technique. The seven extraocular muscles can each be divided into two neuromuscular compartments. The intramuscular nerve-dense regions of the superior, inferior, medial, and lateral rectus and the superior oblique, inferior oblique, and levator palpebrae superioris muscles were positioned at $33.50 \%-72.72 \%$, $40.21 \%-$ $66.79 \%, 37.92 \%-64.51 \%, 31.69 \%-56.01 \%, 26.35 \%-64.98 \%, 40.46 \%-73.20 \%$, and $27.72 \%-66.07 \%$ of the lengths of the muscle bellies, respectively, and the centers of intramuscular nerve dense regions were located at 59.50 \%, 54.18\%, 51.68 \%, 50.08\%, $48.38 \%, 56.49 \%$, and $50.77 \%$ of the length of each muscle belly, respectively. The aforementioned values are the means of the actual values. These results suggest that when the strabismus is corrected with muscle transplantation, the extraocular muscle should be transplanted based on the neuromuscular compartment, which would benefit the function of both donor and recipient muscles. The localization of these nerve dense regions is recommended as an optimal target for the injection of botulinum toxin A to treat strabismus.

KEY WORDS: Extraocular muscles; Intramuscular nerve; Distribution pattern; Neuromuscular compartment; Strabismus.

\section{INTRODUCTION}

The extraocular muscles consist of the superior rectus (SR), inferior rectus (IR), medial rectus (MR), lateral rectus (LR), superior oblique (SO), inferior oblique (IO), and levator palpebrae superioris (LPS) muscles (Frank, 2014). Both eyes can maintain a normal eye position while achieving the goal of binocular single vision. The agonist, antagonist, and synergetic muscles can coordinate their movements in a tacit manner that is indistinguishable from the fine adjustment of all extraocular muscles by the nervous system (Gunton et al. 2015). Hypertension, diabetes, infection, tumor oppression, and ocular trauma can cause extraocular muscle paralysis, eye movement disorders, strabismus, and ptosis. Certain central nervous system diseases such as stroke, brain injury, and other conditions secondary to the spasticity of the extraocular muscles can also cause strabismus (Rowe \& Noonan, 2017).
The clinical injection of botulinum toxin type A (BTX-A) into the motor end plate of antagonistic or agonist muscles to block the release of acetylcholine or to facilitate extraocular muscle transplantation can be used to correct strabismus caused by extraocular muscle paralysis and spasticity (Sanz et al. 2020). The efficacy of BTX-A depends on the proximity of the injection site to the motor endplate band (i.e., the target). Motor endplate staining is limited due to the lack of availability of fresh specimens. Since the location of the intramuscular nerve dense region (INDR) coincides with that of the motor endplate band, the INDR can be used as an alternative target for BTX-A injection (Wang et al. 2020). Whole muscle transplantation can cause loss of function at the donor site (Inal et al. 2020). The function of the donor and recipient regions should be considered simultaneously if a muscle is transplanted (Yan 
et al. 2015), based on the principle of division of neuromuscular compartments (i.e., the innervation range of each independent primary nerve branch in the muscle constitutes one compartment).

Therefore, the purpose of this study was to clearly display the overall distribution pattern of the intramuscular nerves of the extraocular muscles using a modified Sihler's staining technique to explore the division of its neuromuscular compartments, and localize the innervated nerve dense region so as to provide morphological guidance to correct strabismus through the transplantation of the extraocular muscles and the BTX-A injection.

\section{MATERIAL AND METHOD}

Specimens and ethics. We examined 12 formalin-fixed head specimens of Chinese adults (six men and six women) who were aged 35-70 years and had no neuromuscular diseases or ocular muscle diseases. The collection and use of the specimens were approved by the ethics committee of the Zunyi Medical University (Zunyi, China; approval no.: 20201-008).

Gross anatomy. The cranium was opened by removing the Calvaria, sawing above the eyebrow, $1 \mathrm{~cm}$ above auricle, and $1 \mathrm{~cm}$ above the external occipital protuberance. Brain tissue was removed. The top wall of the optic nerve tube and the supraorbital wall were completely severed. The extraocular muscles were fully exposed, and the orbital fascia was removed. We observed the shape, fiber arrangement, and the origin and insertion of theextraocular muscles, along with the source of the nerve muscle branch and its nerve entry point. The extraocular muscles from both sides of each head were completely removed (24 in total).

Sihler's staining. Due to the small extraocular muscle mass, the reagent and processing time were adjusted based on previous experience (Wang et al.). We performed Sihler's staining as follows: (1) pigment removal: we applied 3\% potassium hydroxide and $0.2 \%$ hydrogen peroxide solution to the specimens for 2-3 weeks; (2) decalcification: Sihler's I solution (1 part glacial acetic acid, 2 parts glycerin, 12 parts $1 \%$ chloral hydrate) was used to decalcify the specimens for 2-3 weeks; (3) staining: specimens were stained using Sihler's II solution (1 part Ehrlich dyeing liquid, 2 parts glycerin, and 12 parts $1 \%$ chloral hydrate) for 4 weeks; (4) decolorization: the specimens were placed in Sihler's I solution for 4-10hours; (5) neutralization: the specimens were placed in $0.05 \%$ lithium carbonate solution for 2 hours; and (6) transparency: the specimens were soaked in a glycerin solution while gradually increasing its concentration gradient (i.e., $40 \%, 60 \%, 80 \%, 100 \%$ ) for 1 week. The distribution of the intramuscular nerves was observed under an X-ray lamp, after which it was photographed. We also drew a pattern diagram based on our findings.

\section{Localization of theintramuscular nerve-dense regions} and the center of the intramuscular nerve dense region. We framed the dense region of intramuscular nerve branches using Photoshop CC 2020 (Adobe Systems Inc., San Jose, CA, USA). The frame was then double-clicked to select the region. The pop-up center point was designated as the center of the intramuscular nerve dense region (CINDR) and marked. The positions of the INDR and CINDR in the length of the muscle belly were calculated by percentage, respectively (i.e., the percentage position on the length of the muscle belly was calculated as the longitudinal distance between the nearest origin of the muscle fiber and the INDR or CINDR divided by the length of the muscle belly). The region of the irregular INDR was measured using a computer-aided design software (Autodesk; San Rafael, CA, USA). Finally, the map of the nerve distribution pattern was restored to the corresponding position of the muscle, based on the corresponding proportion.

Statistical analyses. We used SPSS18.0 software (IBM, Chicago, IL, USA) for data analyses. The paired test was used to compare the data between the right and left muscles, and the independent sample T-test was used to compare data between men and women. The statistical significance was set at $\mathrm{P}<0.05$.

\section{RESULTS}

Gross anatomy observation. Besides the LPS, all six of the other extraocular muscles were attached to the eyeball in a conical shape. After the oculomotor nerve entered the orbit from the supraorbital fissure, it divided into a thin superior branch and thick inferior branch. The superior branch of the oculomotor nerve obliquely extended forward and upward and diverged into the SR branch at 1/3 of the deep surface near the origin of the SR; it then continued to extend inward and upward to reach the middle deep surface of the LPS muscle above it and entered the muscle. The MR, IR, and IO branches, which entered into the muscle laterally from the origin of the muscle belly, deep from the origin of the muscle belly, and laterally from the middle part of the muscle belly, respectively, all stemmed from the inferior branch of the oculomotor nerve. The abducens nerve entered the orbit from the supraorbital fissure, after passing through the ring of the common tendon. It was near the medial surface 
of the LR and entered the muscle at $1 / 3$ of the length of the muscle's origin. After entering the orbit from the superior orbital fissure, the trochlear nerve passed over the SR and the LPS, traveled medially anteriorly, and entered the muscle on the lateral surface at $1 / 3$ of the origin of the SO muscle (Fig. 1).

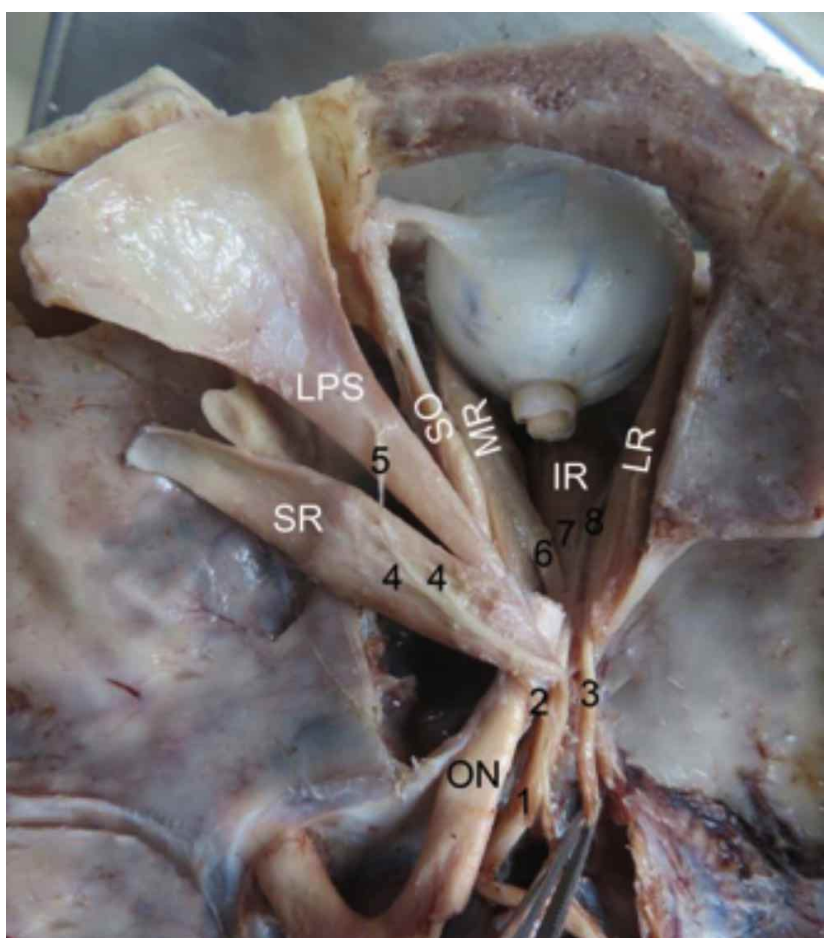

Fig. 1 Gross anatomy of the right extraocular muscle and its innervation. $\mathrm{SR}=$ superior rectus, $\mathrm{LPS}=$ levator palpebrae superioris, $\mathrm{SO}=$ superior oblique, $\mathrm{MR}=$ medial rectus, $\mathrm{IR}=$ inferior rectus, $\mathrm{LR}=$ lateral rectus, $\mathrm{ON}=$ optic nerve. $1=$ oculomotor nerve, $2=$ trochlear nerve, $3=$ abducens nerve, $4=$ superior rectus branch of the oculomotor nerve, $5=$ levator muscle branch of the oculomotor nerve, $6=$ medial rectus branch of the oculomotor nerve, $7=$ inferior rectus branch of the oculomotor nerve, $8=$ inferior oblique branch of the oculomotor nerve.

Observation of Sihler's staining. The muscle appeared intact and was light purple in color, while the nerve branches were black. The intramuscular nerve branches were Yshaped. The dense arborized branches were mostly distributed across the entire width of the center of the muscle and communicated with one other to form an intramuscular nerve plexus. The distribution pattern did not differ significantly between the left and right sides of the cranium or between men and women.

Superior rectus muscle. Two primary branches (i.e., the medial and lateral branches) were visible before the entry of the SR muscle's nerve branches. After entering the muscle, the lateral branch first divided into two secondary branches and then diverged into 4-7 tertiary branches. It then extended to the insertion in an arborized fashion. Overlapping regions existed among its small branches, which primarily innervated the lateral half of the muscle belly. After the medial branch entered the muscle, 4 to 6 secondary branches were visible, which were distributed throughout the medial half of the muscle belly. The arborized branches of these primary branches communicated intramuscularly and were densely distributed between $33.50 \% \pm 0.86 \%$ and $72.72 \% \pm$ $1.23 \%$ of the muscle belly length, forming a pear-shaped INDR. The size of the region and the position of the central point of the dense region on the length of the muscle belly are shown in Tables I and II. The muscle could be divided into two neuromuscular compartments (Fig. 2).

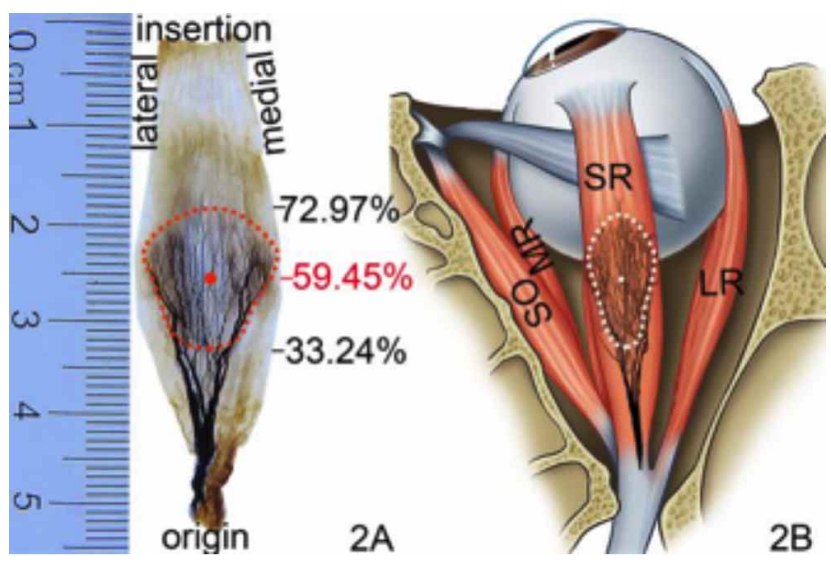

Fig. 2 Distribution and location of the intramuscular nerves in the right superior rectus muscle. 2A: Sihler's staining (inferior view). The frame of red squares indicates the intramuscular nerve dense region (INDR), and the red dot indicates the center of the intramuscular nerve dense region (CINDR). Ruler: $\mathrm{cm}$. 2B: The position of the INDR and CINDR on the skeleton. The irregular frame of white dots indicates the INDR, and the central white dot indicates the CINDR.SR = superior rectus, $\mathrm{SO}=$ superior oblique, $\mathrm{MR}=$ medial rectus, $\mathrm{LR}=$ lateral rectus.

Inferior rectus muscle. The nerve branches of the IR split into two primary nerve branches at $1 / 4$ of the length from the origin of the muscle, and entered the muscle after approximately $0.5 \mathrm{~cm}$. Among the branches, the lateral primary branch was slightly thicker and could further be divided into two secondary branches at a position of $1 / 3$ from the origin of the muscle. Then, the secondary branches divided into arborized branches in a Y-shaped manner to dominate the lateral half of the muscle belly. The medial primary branch was slightly thinner and branched into secondary branches earlier, dominating the medial muscle fibers. The arborized branches of the two primary branches overlapped at the center of the muscle. The terminals of these arborized branches formed a chestnut-shaped INDR at a 
position of $40.21 \% \pm 0.75 \%$ to $66.79 \% \pm 0.83 \%$ of the muscle belly length. The size of the region and the position of the central point of the dense region on the length of the muscle belly are shown in Tables I and II. The muscle could be divided into two neuromuscular compartments (Fig. 3).

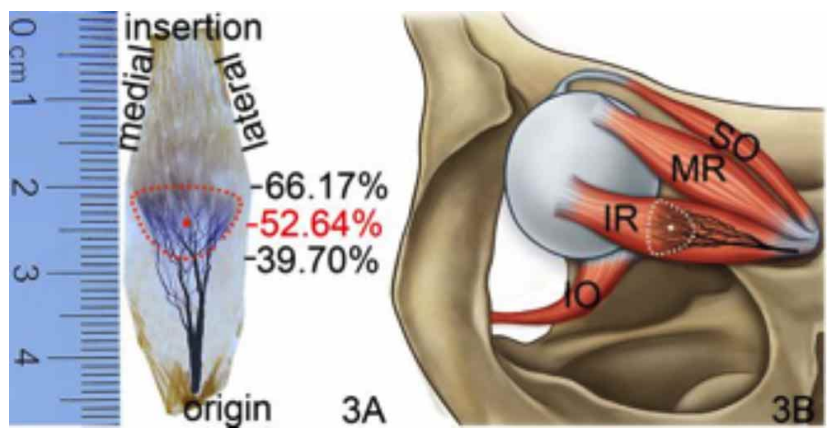

Fig. 3 Distribution and location of intramuscular nerves in the right inferior rectus muscle.3A: Sihler's staining (superior view). Ruler: $\mathrm{cm}$. The irregular frame of red dots represents the INDR, and the central red dot represents the CINDR. 3B: The position of INDR and CINDR on the skeleton. The irregular frame of white dots represents the INDR, and the central white dot represents the CINDR. IR = inferior rectus, $\mathrm{SO}=$ superior oblique, $\mathrm{MR}=$ medial rectus, $\mathrm{IO}=$ inferior oblique.

Medial rectus muscle. The MR nerve branch diverged into two primary nerve branches immediately after entering the muscle. Each primary nerve branch was further divided into three to four secondary nerve branches. The arborized branches stemming from these secondary nerve branches were distributed across the middle of the muscle belly, and the branches communicated with one other while being positioned at $37.92 \% \pm 0.56 \%$ to $64.51 \% \pm 0.90 \%$ of the muscle belly, forming a chestnut-shaped INDR. The size of the region and the position of the central point of the dense region on the length of the muscle belly are shown in Tables I and II. The muscle can be divided into two neuromuscular compartments (Fig. 4).
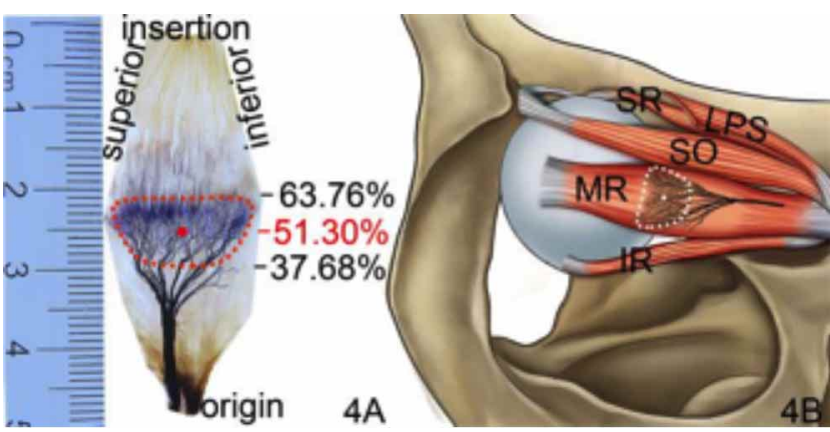

Fig. 4 Distribution and location of intramuscular nerves in the right medial rectus muscle. 4A: Sihler's staining (lateral view). Ruler: $\mathrm{cm} .4 \mathrm{~B}$ : The position of INDR and CINDR on the skeleton.
Lateral rectus muscle. When the abducens nerve entered the muscle, it immediately split into two primary nerve branches (i.e., the superior and inferior branches). Each primary nerve branch diverged into three to four secondary nerve branches toward the insertion of the muscle. The secondary nerve branches split into small, arborized branches that could communicate. The arborized branches formed an overlapping innervation region at the center of the muscle belly and formed a chestnut-shaped nerve dense region at a position between $31.69 \% \pm 0.67 \%$ and $56.01 \% \pm 0.63 \%$ of the muscle belly length. The size of the region and the position of the central point of the dense region on the length of the muscle belly are shown in Tables I and II. The muscle could be divided into two neuromuscular compartments (Fig. 5).

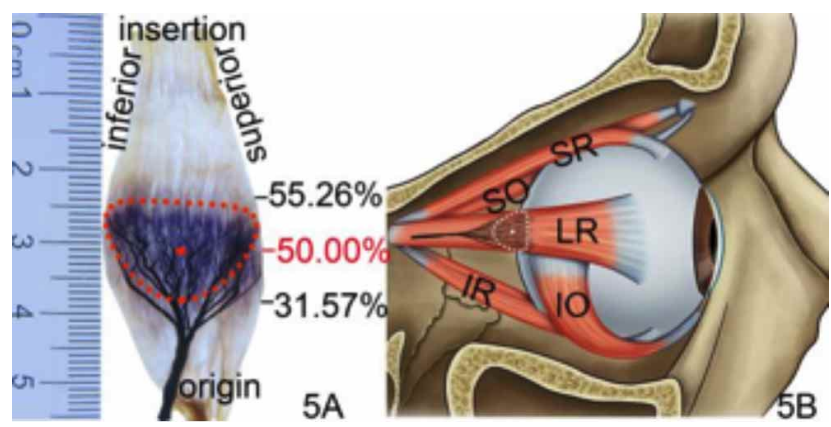

Fig. 5 Distribution and location of the intramuscular nerves in the right lateral rectus muscle. 5A: Sihler's staining (medial view). Ruler: cm.5B: The position of INDR and CINDR on the skeleton.

Superior oblique muscle. The trochlear nerve entered the SO muscle throughits lateral surface. After entering the muscle, it often divided into two primary nervebranches (i.e., superiorand inferior branches). It ran obliquely inward and upward. Arborized branches began at approximately $1 / 3$ of the length of the muscle belly. The inferior branch was thick, with 2-3 secondary branches, and primarily innervated the inferior half of the muscle belly (breadth wise). The superior branch was small and dominated the superior half of the muscle belly (breadth wise). Its branches communicated with those of the inferior branch. The arborized branches formed an oval-shaped INDR positioned between $26.35 \%$ $\pm 0.28 \%$ and $64.98 \% \pm 0.13 \%$ of the length of the muscle belly. The size of the region and the position of the CINDR on the length of the muscle belly are shown in Tables I and II. The muscle could be divided into two neuromuscular compartments (Fig. 6).

Inferior oblique muscle. The IO nerve branch arose from the oculomotor nerve, which ranfrom the posterior to the anterior. It entered the muscle laterally in the middle of the muscle belly and divided into superior and inferior primary nerve branches after entering the muscle. Several branches diverged 
upward and forward from the superior branch, and the branches anastomosed with one other to form a net. The arborized branches of the superior and inferior branches were clustered into a rectangular INDR positioned between $40.46 \% \pm 0.70 \%$ and $73.20 \% \pm 0.67 \%$ of the muscle belly length. The size of the region and the position of the CINDR on the muscle belly length are shown in Tables I and II. The muscle could be divided into two neuromuscular compartments (Fig. 7).

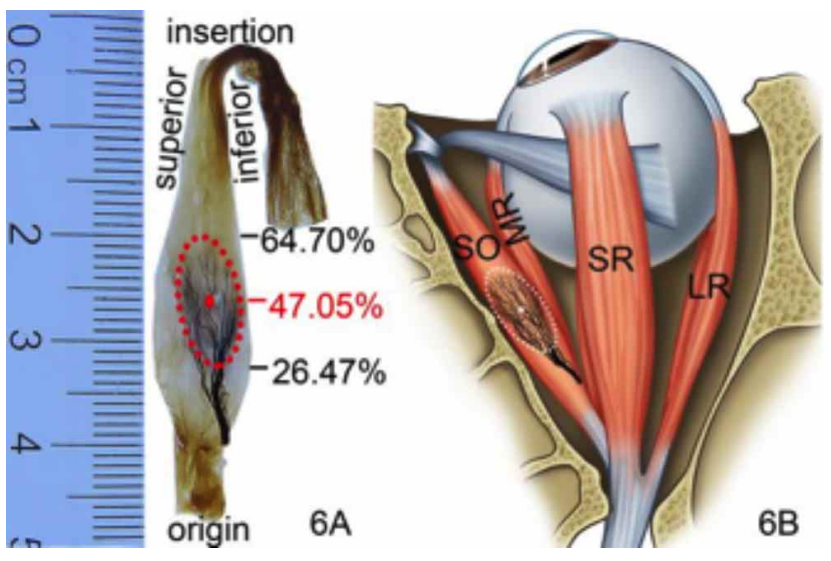

Fig. 6 Distribution and location of the intramuscular nerves in the right superior oblique muscle. 6A: Sihler's staining (lateral view). Ruler: cm. 6B: The position of INDR and CINDR on the skeleton.

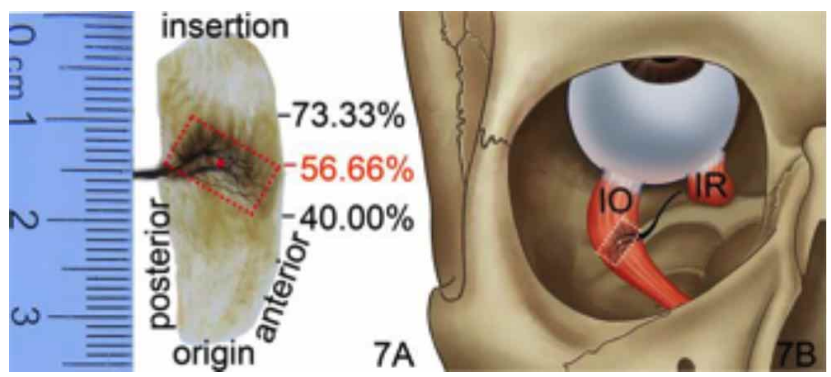

Fig. 7 Distribution and location of intramuscular nerves in the right inferior oblique muscle. 7A: Sihler's staining (lateral view). Ruler: $\mathrm{cm}$. 7B: The position of INDR and CINDR on the skeleton.
Levator palpebrae superioris muscle. After the LPS nerve branch entered the muscle, it divided into two primary nerve branches: medial and lateral. The medial branch was relatively thin and ran forward and inward. It sent out small nerve branches at a position between $50.77 \% \pm 0.67 \%$ and $66.07 \% \pm 0.74 \%$ of the muscle belly length that innervated the medial $1 / 3$ of the muscle. The lateral branch was relatively thick and ran forward and laterally. It further diverged into arborized branches and innervated the lateral $2 / 3$ of the muscle. Some branches extended into the tendon at the end of the insertion. The branches of the two primary branches were anastomotic. The arborized branches were most dense at $27.22 \% \pm 0.32 \%$ to $66.07 \pm 0.74 \%$ of the muscle belly length and formed an oval nerve dense region. The size of the INDR and the position of the CINDR on the length of the muscle belly are shown in Tables I and II. The muscle could be divided into two neuromuscular compartments (Fig. 8).

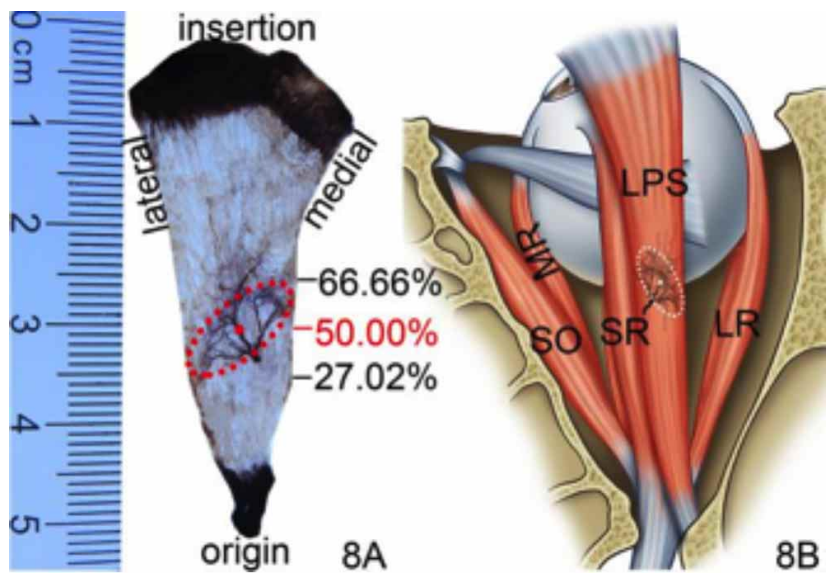

Fig. 8 Distribution and location of intramuscular nerves in the right levator palpebrae superioris muscle. 8A: Sihler's staining (lateral view). Ruler: $\mathrm{cm}$. $8 \mathrm{~B}$ : The position of INDR and CINDR on the skeleton.

Based on the analysis of the 24 extraocular muscles, the CINDRs of the SR, IR, MR, LR, SO, IO, and LPS muscles were positioned at $59.50 \% \pm 0.67 \%, 54.18 \% \pm$

Table I. Comparison of the INDR area and the percentage position of the CINDR on the muscle belly length between the left and right sides $(\bar{x} \pm \mathrm{s})$.

\begin{tabular}{|c|c|c|c|c|c|c|c|c|}
\hline \multirow{2}{*}{ Muscle } & \multicolumn{4}{|c|}{ Area of INDR $\left(\mathrm{cm}^{2}\right)$} & \multicolumn{4}{|c|}{ Position of CINDR on muscle belly length (\%) } \\
\hline & Left $(n=12)$ & Right $(n=12)$ & $t$ & $\bar{P}$ & Left $(n=12)$ & Right $(n=12)$ & $\bar{t}$ & $\bar{P}$ \\
\hline SR & $1.41 \pm 0.08$ & $1.45 \pm 0.07^{\Delta}$ & -1.173 & 0.266 & $59.13 \pm 0.82$ & $59.50 \pm 0.67^{\Delta}$ & -1.133 & 0.281 \\
\hline IR & $1.29 \pm 0.08$ & $1.27 \pm 0.06^{\Delta}$ & 0.561 & 0.586 & $53.94 \pm 1.09$ & $54.18 \pm 0.77^{\Delta}$ & -0.572 & 0.579 \\
\hline MR & $1.30 \pm 0.12$ & $1.30 \pm 0.09^{\Delta}$ & 0.029 & 0.978 & $51.31 \pm 0.61$ & $51.68 \pm 0.87^{\Delta}$ & -1.008 & 0.335 \\
\hline LR & $1.62 \pm 0.16$ & $1.71 \pm 0.17^{\Delta}$ & -1.173 & 0.266 & $50.09 \pm 0.26$ & $50.08 \pm 0.34^{\Delta}$ & 0.077 & 0.094 \\
\hline SO & $0.63 \pm 0.09$ & $0.66 \pm 0.06^{\Delta}$ & -1.114 & 0.289 & $48.19 \pm 0.71$ & $48.38 \pm 0.66^{\Delta}$ & -0.561 & 0.586 \\
\hline IO & $0.69 \pm 0.10$ & $0.72 \pm 0.14^{\Delta}$ & -0.484 & 0.638 & $56.20 \pm 0.55$ & $56.49 \pm 0.74^{\Delta}$ & -0.931 & 0.372 \\
\hline LPS & $0.75 \pm 0.17$ & $0.80 \pm 0.18^{\Delta}$ & -0.482 & 0.639 & $50.60 \pm 0.50$ & $50.77 \pm 0.67^{\Delta}$ & -0.551 & 0.592 \\
\hline
\end{tabular}

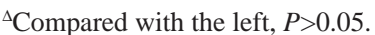


$0.77 \%, 51.68 \% \pm 0.87 \%, 50.08 \% \pm 0.34 \%, 48.38 \% \pm$ $0.66 \%, 59.49 \% \pm 0.74 \%$, and $50.77 \% \pm 0.67 \%$ of the muscle belly length, respectively; the INDRs were $1.45 \pm$ $0.07 \mathrm{~cm}^{2}, 1.27 \pm 0.06 \mathrm{~cm}^{2}, 1.30 \pm 0.09 \mathrm{~cm}^{2}, 1.71 \pm 0.17 \mathrm{~cm}^{2}$,
$0.66 \pm 0.06 \mathrm{~cm}^{2}, 0.72 \pm 0.14 \mathrm{~cm}^{2}$, and $0.80 \pm 0.18 \mathrm{~cm}^{2}$ in length, respectively. We did not observe significant differences between the left and right sides or between men and women $(\mathrm{P}>0.05)$.

Table II. Comparison of the INDR area and the percentage position of the CINDR on the muscle belly length between men and women $(\bar{x} \pm \mathrm{s})$

\begin{tabular}{|c|c|c|c|c|c|c|c|c|}
\hline \multirow[b]{2}{*}{ Muscle } & \multicolumn{4}{|c|}{ Area of INDR $\left(\mathrm{cm}^{2}\right)$} & \multicolumn{4}{|c|}{ Position of CINDR on muscle belly length (\%) } \\
\hline & $\operatorname{Men}(n=6)$ & W omen $(n=6)$ & $t$ & $P$ & Men $(n=6)$ & Women $(n=6)$ & $t$ & $P$ \\
\hline SR & $1.48 \pm 0.05$ & $1.43 \pm 0.08^{\Delta}$ & 1.490 & 0.167 & $59.38 \pm 0.89$ & $59.79 \pm 0.54 \Delta$ & -0.950 & 0.365 \\
\hline IR & $1.24 \pm 0.09$ & $1.29 \pm 0.07^{\Delta}$ & -1.015 & 0.334 & $54.60 \pm 0.81$ & $54.47 \pm 0.85 \Delta$ & 0.270 & 0.792 \\
\hline MR & $1.19 \pm 0.03$ & $1.24 \pm 0.07^{\Delta}$ & -1.514 & 0.161 & $51.81 \pm 1.04$ & $51.17 \pm 0.85 \Delta$ & 1.178 & 0.266 \\
\hline LR & $1.51 \pm 0.11$ & $1.59 \pm 0.11^{\Delta}$ & -1.336 & 0.211 & $50.07 \pm 0.26$ & $49.95 \pm 0.44 \Delta$ & 0.563 & 0.586 \\
\hline $\mathrm{SO}$ & $0.60 \pm 0.11$ & $0.67 \pm 0.07^{\Delta}$ & -1.279 & 0.230 & $47.64 \pm 0.42$ & $48.01 \pm 0.71 \Delta$ & -1.100 & 0.297 \\
\hline IO & $0.64 \pm 0.11$ & $0.77 \pm 0.11^{\Delta}$ & -2.037 & 0.069 & $55.74 \pm 0.33$ & $56.12 \pm 0.56 \Delta$ & -1.423 & 0.185 \\
\hline LPS & $0.87 \pm 0.15$ & $0.74 \pm 0.18^{\Delta}$ & 1.399 & 0.192 & $50.72 \pm 0.52$ & $50.59 \pm 0.61 \Delta$ & 0.402 & 0.70 \\
\hline
\end{tabular}

${ }^{\Delta}$ Compared with men, $P>0.05$.

\section{DISCUSSION}

The intramuscular nerve endings of human extraocular muscles have been the focus of several research papers. Daniel (1946) discovered several spiral nerve endings in the human extraocular muscles using paraffin silver staining as early as 1946. In a later study, Ruskell (1984) applied acetylcholinesterase technology to stain human extraocular muscles and found that approximately $5 \%$ of the motor endplates also have spiral terminals based on the results of light and electron microscope observations. Ruskell believed that these terminals were not used to monitor the contraction of muscle fibers but rather to attain unconventional contraction speed. The composition of acetylcholine receptor subunits was recently studied with immunohistochemistry techniques. It was found that one fiber of human extraocular muscle may be innervated by multiple neurons (Liu \& Domellöf, 2018). Many studies have focused on the nerve endings in the extraocular muscles; however, the specific location of the whole motor endplate band of the extraocular muscles has not been described. In the middle to late 20th century, some scholars proposed the application of a local injection of denervating drugs (e.g., BTX-A) rather than surgery to treat strabismus, which prevents the occurrence of surgical complications. Its mechanism of action is the block the release of acetylcholine at the motor endplate, inhibition of muscle excitation, and coordination of the dynamic balance between muscles to treat strabismus (Rowe \& Noonan). Studying the overall distribution pattern of intramuscular nerves can provide guidance for locating alternative targets of BTX-A and transplantation of extraocular muscle compartments for the treatment of strabismus. In this study, Sihler's staining was used to systematically reveal the distribution pattern of the intramuscular nerves and the position of the INDR (i.e., the alternative target of BTX-A) in each extraocular muscle. The study also explored the compartment that would be of the greatest morphological significance for chemical denervation and surgical muscle transplantation to facilitate the correction of strabismus.

Some studies have analyzed the intramuscular nerves of the four rectus muscles, as well as those of the IO and LPS muscles (Haladaj, 2020; Haladaj et al. 2020a; Haladaj et al. 2020b; Shin, 2020). These studies primarily described the distribution pattern of the intramuscular nerve branches, while only the location of the BTX-A injection site in the nerve dense region of the LR muscle was described. The nerve distribution patterns in the SO muscle have not yet been studied. In the current study, we systematically observed the nerve distribution patterns of the seven extraocular muscles and noted that the intramuscular nerve distribution patterns of the four rectus, IO, and LPS muscles were basically consistent with those described by Haladaj and Shin. However, we found that the anterior end of the nerve dense region of the LR muscle described by Shin $e t$ al. (2020) was, on average, $24.8 \mathrm{~mm}$ behind the muscle insertion, suggesting that BTX-A should be injected approximately $25 \mathrm{~mm}$ behind the muscle insertion. The absolute distance may be affected by individual differences, thereby causing inaccurate positioning. The suggested blocking point is not in the nerve dense region, but in front of it. The infiltration of the drug into the front of the dense region is ineffective. This study improved the localization of the CINDRs of all muscle to reduce the effect of individual differences. Data are expressed as percentages. 
A BTX-A injection can temporarily paralyze the agonist muscle in the extraocular muscle and reduce the tension, whereas the tension of the antagonistic muscle increases in relation, which can correct the eye position without changing the anatomical position of the muscle. At present, BTX-A injection is primarily used to treat concomitant strabismus, paralytic esotropia, horizontal strabismus, or vertical strabismus. It can also be used for overcorrection and under-correction after strabismus surgery (Rowe \& Noonan). Some data demonstrate that the injection dose of BTX-A depends on the degree of strabismus. One can safely use 2.5-5.0 units of BTX-A to treat this condition (Rowe \& Noonan). The effect depends on the distance between the target and the motor endplate. If the target position is $5 \mathrm{~mm}$ away from the motor end plate, the effect will be reduced by $50 \%$ (Parratte et al. 2002). If a large dose or multipoint injection is administered, antibodies will be produced, causing the drug to spread to the surrounding muscle tissue, resulting in unnecessary complications (Shaari \& Sanders, 1993). For example, when BTX-A is injected into the orbicularis oculi muscle to treat blepharospasm, BTX-A may infiltrate the LPS muscle, thereby causing blepharoptosis (Tejedor \& Rodríguez, 1998). When treating upper eyelid contracture, large doses and multiple injections of BTX-A can cause SR paralysis (Naik et al. 2008). Therefore, the accurate injection ofdrugs into the target site is essential.

One unit of BTX-A can infiltrate an area of 1.5-3.0 $\mathrm{cm}^{2}$, and 2.5-5.0 units can diffuse through an area of up to $4.5 \mathrm{~cm}^{2}$ (Borodic et al. 1994). Based on this conclusion and the INDR region measured in this study, the actual dose required for the $\mathrm{SR}, \mathrm{IR}, \mathrm{MR}$, and LR muscles is 1 unit, whereas the dose for the SO, IO, and LPS muscles is only 0.5 units. If the location is accurate, the dosage of BTX-A can be reduced, thereby reducing the risk of complications.

Surgery is a common method for treating strabismus, which can be corrected by muscle displacement, retreat, or resection to strengthen or weaken the muscles (Eraslan et al. 2015). For example, SR and IR muscle transposition can be used to treat esotropia (Sener et al. 2019). MR and LR muscle transposition can be used to treat recurrent and residual strabismus (Kannam et al. 2021). Transposition of the $\mathrm{SO}$ muscle can be used to treat oculomotor paralysis (Eraslan et al.). Anterior transposition of the IO muscle can be used to treat IR hypoplasia and SO paralysis (Singh et al. 2019; Saxena et al. 2017). In addition, the LPS muscle can be partially transplanted to treat SR paralysis (Stallard, 1952). However, transposition of the whole muscle risks the loss of function at the donor site and the development of new types of strabismus. Therefore, this study recommends compartmentalized muscle transplantation while considering the functions of the donor and recipient regions (Yan et al.). In this study, we found that all seven extraocular muscles had divided neuromuscular compartments. Based on the muscle strength and the amplitude of the recipient region, more matching neuromuscular compartments in clinical muscle transplantation can be selected. This action allows the recipient region to have normal innervation and the best recovery of function while avoiding complete damage to the donor region's function. However, it is worth noting that the IO muscle should not be split longitudinally into two muscle compartments because its nerves run laterally from posterior to anterior in the middle of the muscle.

This is the first study to our knowledge to systematically reveal the overall distribution pattern of the intramuscular nerves in seven extraocular muscles, explore neuromuscular compartments, and localize the INDR and CINDRs on the length of the muscle belly. Our results suggest that, when strabismus is corrected with muscle transplantation, the extraocular muscle should be transplanted based on neuromuscular compartments, which would benefit the function of the donor and recipient muscles. The localization of these nerve dense regions is recommended to provide optimal targets for BTX-A injection to treat strabismus. However, this study has some limitations, such as a lack of comparison between racial differences and are natively small sample size. In addition, the efficacy of this approach needs to be verified in a clinical setting.

\section{ACKNOWLEDGMENTS}

This work were supported by the National Natural Science Foundation of China (No. 31660294, 31540031) and Science and technology projects of Guizhou Province (No.ZK[2021]-115)

ZHOU, G.; ZHOU, J.; WANG, D. \& YANG, S. Patrón de distribución general de los nervios intramusculares de los músculos extraoculares y sus implicaciones clínicas. Int. J. Morphol., 39 (5):1412-1419, 2021

RESUMEN: El objetivo de este estudio fue revelar el patrón de distribución de los nervios intramusculares de cada músculo extraocular y, proporcionar una guía morfológica para la selección del compartimento neuromuscular durante el trasplante de músculo extraocular, y la localización de la inyección de toxina botulínica A para corregir el estrabismo. Estudiamos 12 muestras de cabezas de individuos chinos fijadas en formalina. Se extrajeron los músculos extraoculares de ambos lados de cada cabeza y, se utilizó una técnica de tinción de Sihler modificada para revelar el patrón de distribución general de los nervios intramusculares. Observamos una región densa en nervios intramusculares forma- 
da por los ramos intramusculares en los músculos recto superior semitransparente, recto inferior, recto medial, recto lateral, oblicuo superior, oblicuo inferior y elevador del párpado superior con técnica de tinción de Sihler. Los siete músculos extraoculares se pueden dividir cada uno en dos compartimentos neuromusculares. Las regiones intramusculares densamente nerviosas de los músculos recto superior, inferior, medial y lateral y los músculos oblicuo superior, oblicuo inferior y elevador del párpado superior se colocaron en $33,50 \%-72,72 \%, 40,21 \%-66,79 \%, 37,92 \%-64,51 \%, 31,69 \%$ $56,01 \%, 26,35 \%-64,98 \%, 40,46 \%-73,20 \%$ y $27,72 \%-66,07 \%$ de las longitudes de los vientres musculares, respectivamente, y los centros de las regiones densamente nerviosas intramusculares se ubicaron en 59,50\%, 54,18 \% , 51,68 \%, 50,08 \%, 48,38\%, 56,49\% y $50,77 \%$ de la longitud de cada vientre muscular, respectivamente. Los valores antes mencionados son medios de los valores reales. Estos resultados sugieren que cuando el estrabismo se corrige con trasplante de músculo, el músculo extraocular debe trasplantarse en función del compartimento neuromuscular, lo que beneficiaría la función tanto de los músculos donantes como receptores. Se recomienda la localización de estas regiones densas en nervios, como un objetivo óptimo para la inyección de toxina botulínica A para tratar el estrabismo.

PALABRAS CLAVE: Músculos extraoculares; Nervio intramuscular; Patrón de distribución; Compartimento neuromuscular; Estrabismo.

\section{REFERENCES}

Borodic, G. E.; Ferrante, R.; Pearce, L. B. \& Smith, K. Histologic assessment of dose-related diffusion and muscle fiber response after therapeutic botulinum A toxin injections. Mov. Disord., 9(1):31-9, 1994.

Daniel, P. Spiral nerve endings in the extrinsic eye muscles of man. J. Anat., 80(4):189-93, 1946.

Eraslan, M.; Cerman, E.; Onal, S. \& Ogut, M. S. Superior oblique anterior transposition with horizontal recti recession-resection for total thirdnerve palsy. J. Ophthalmol., 2015:780139, 2015.

Frank, H. Atlas of human anatomy. $6^{\text {th }}$ ed. USA, Saunders an imprint of Elsevier Inc., pp. 85-88, 2014.

Gunton, K. B.; Wasserman, B. N. \& DeBenedictis, C. Strabismus. Prim Care., 42(3):393-407, 2015.

Haadaj, R. Comparison of lateral and medial rectus muscle in human: an anatomical study with particular emphasis on morphology, intramuscular innervation pattern variations and discussion on clinical significance. Surg. Radiol. Anat., 42(5):607-16, 2020.

Haladaj, R.; Polguj, M. \& Tubbs, R. S. Comparison of the Superior and Inferior Rectus Muscles in Humans: An Anatomical Study with Notes on Morphology, Anatomical Variations, and Intramuscular Innervation Patterns. Biomed. Res. Int., 2020:9037693, 2020a.

Haladaj, R.; Wysiadecki, G. \& Tubbs, R. S. Intramuscular innervation of the lateral rectus muscle evaluated using Sihler's staining technique: Potential application to strabismus surgery. Clin Anat., 33(4):585-91, 2020 b.

Inal, A.; Gokyigit, B.; Ocak, O. B.; Aygit, E. D. \& Celik, S. Vertical rectus muscle union combined with lateral rectus plication for complete abducens nerve palsy. Int. Ophthalmol., 40(2):423-9, 2020.

Kannam, M.; Sutraye, J.; Kapoor, R.; Tibrewal, S.; Kekunnaya, R. \& Sachdeva, V. Horizontal rectus muscle transplantation for recurrent and residual strabismus. Strabismus, 29(1):42-50, 2021.
Liu, J. X. \& Domellöf, F. P. A Novel Type of Multiterminal Motor Endplate in Human Extraocular Muscles. J. Invest. Ophthalmol. Vis. Sci., 59(1):539-48, 2018.

Naik, M. N.; Gangopadhyay, N.; Fernandes, M.; Murthy, R. \& Honavar, S. G. Anterior chemodenervation of levator palpebrae superioris with botulinum toxin type-A (Botox) to induce temporary ptosis for corneal protection. Eye (Lond)., 22(9):1132-6, 2008.

Parratte, B.; Tatu, L.; Vuillier, F.; Diop, M. \& Monnier, G. Intramuscular distribution of nerves in the human triceps surae muscle: anatomical bases for treatment of spastic drop foot with botulinum toxin. Surg. Radiol. Anat., 24(2):91-6, 2002.

Rowe, F. J \& Noonan, C. P. Botulinum toxin for the treatment of strabismus. Cochrane Database Syst. Rev., 3(3):CD006499, 2017.

Ruskell, G. L. Spiral nerve endings in human extraocular muscles terminate in motor end plates. J. Anat.,139(1):33-43, 1984.

Sanz, P. M.; Osuna, V.; Gómez de Liaño Sánchez, P. \& Torres, H. Surgical treatment for small-angle vertical strabismus. Strabismus, 28(4):181$5,2020$.

Saxena, R.; Sharma, M.; Singh, D. \& Sharma, P. Anterior and nasal transposition of inferior oblique muscle in cases of superior oblique palsy. J. AAPOS., 21(4):282-5, 2017.

Sener, E. C.; Yilmaz, P. T. \& Fatihoglu, Ö. U. Superior or inferior rectus transposition in esotropic Duane syndrome: a longitudinal analysis. $J$. AAPOS., 23(1):21.e1-e7, 2019.

Shaari, C. M. \& Sanders, I. Quantifying how location and dose of botulinum toxin injections affect muscle paralysis. Muscle Nerve, 16(9):964-9, 1993.

Shin, H. J.; Lee, S. H.; Ha, T. J.; Song, W. C. \& Koh, K. S. Intramuscular nerve distribution of the inferior oblique muscle. Curr. Eye Res., 45(2):215-20, 2020.

Singh, A.; Agrawal, A.; Mittal, S. K.; Kumar, B.; Rana, K. M. \& Verma, R. Anterior transposition of inferior oblique for inferior rectus muscle aplasia. Taiwan J. Ophthalmol., 13, 9(4):284-7, 2019.

Stallard, H. B. Partial transplantation of the levator palpebrae superioris in superior rectus palsy. Br. J. Ophthalmol., 36(1):26-8, 1952.

Tejedor, J. \& Rodríguez, J. M. Retreatment of children after surgery for acquired esotropia: reoperation versus botulinum injection. $B r . J$. Ophthalmol., 82(2):110-4, 1998.

Wang, J.; Wang, Q.; Zhu, D.; Jiang, Y. \& Yang, S. Localization of the center of the intramuscular nerve dense region of the medial femoral muscles and the significance for blocking spasticity. J. Ann. Anat., 231:151529, 2020.

Yan, X.; Liu, J.; Liu, B.; Liu, Z.; Pan, Y.; Jia, X.; Zhang, Y. \& Shao, J. [Procedure of reconstruting transverse arch of the forefoot by transferring tendons for correcting hallux valgus]. Zhongguо Хіи Fu Chong Jian Wai Ke Za Zhi., 29(4):412-5, 2015.

Corresponding author:

Shengbo Yang

Department of Anatomy

Zunyi Medical University

6 West Xufu Road

Xinpu Developing Zones

Zunyi 563099

CHINA

E-mail: yangshengbo8205486@163.com

Received: 06-07-2021

Accepted: 27-07-2021 